01.1;08.3;09.4

\title{
Моделирование ангармонических блоховских осцилляций: численные проблемы и нелинейные эффекты
}

\author{
() К.А. Иванов ${ }^{1,2}$, Е.И. Гиршова ${ }^{2}$, М.А. Калитеевский ${ }^{1-3}$ \\ ${ }^{1}$ Санкт-Петербургский национальный исследовательский Академический университет им. Ж.И. Алфёрова РАН, \\ Санкт-Петербург, Россия \\ ${ }^{2}$ Университет ИТМО, Санкт-Петербург, Россия \\ ${ }^{3}$ Физико-технический институт им. А.Ф. Иофффе РАН, Санкт-Петербург, Россия \\ E-mail: kivanov1992@gmail.com
}

Поступило в Редакцию 30 июня 2020г.

В окончательной редакции 28 сентября 2020 г.

Принято к публикации 28 сентября 2020 г.

\begin{abstract}
Представлен устойчивый быстрый численный метод для расчета комплексных энергий и волновых функций носителей в одномерных электрически смещенных периодических структурах. При помощи этого метода исследованы оптические переходы в сверхрешетке (блоховские осцилляции). Показано, что вероятности переходов не подчиняются линейной зависимости от приложенного поля при достаточно больших его значениях. В этом случае переходы на двойной и тройной блоховской частоте могут быть более интенсивными, чем на основной частоте. В сверхрешетке с расщепленной минизоной наблюдается аналогичная картина, причем нелинейность заметнее при сильном расщеплении.
\end{abstract}

Ключевые слова: ангармонические блоховские осцилляции, квазистационарные состояния, штарковская локализация, численное моделирование излучения.

DOI: 10.21883/PJTF.2021.01.50458.18441

Явление блоховских осцилляций (БО), т.е. движения электрона в периодическом потенциале при наличии приложенного поля, было описано в начале прошлого века с использованием квазиклассической теории $[1]$, затем получило квантовое объяснение благодаря формализму функций Ванье [2] и позже наблюдалось экспериментально [3]. Квантовая теория гласит, что при приложении поля к сверхрешетке разрешенная минизона в ней расщепляется на систему эквидистантных локализованных состояний (штарковскую лестницу, ШЛ), расстояние между которыми $\delta E$ соответствует блоховской частоте $\omega_{\mathrm{B}}$ и зависит от периода потенциала $d$ и приложенного поля $F$ как $\delta E=\hbar \omega_{\mathrm{B}}=e F d$.

В настоящее время БО привлекают определенное внимание, при этом теоретически [4] и экспериментально [5] рассматривались их нелинейные свойства, a в нашей предыдущей работе было показано, что при строгом квантовом рассмотрении между уровнями ШЛ возможны излучательные переходы на кратных блоховских частотах [6]. БО привлекательны для различных технических приложений [7], однако сложности моделирования ограничивают темп исследований. Таким образом, целесообразно разработать численные методы, позволяющие максимально быстро моделировать произвольные электрически смещенные периодические структуры.

Основная сложность моделирования БО заключается в том, что состояния ШЛ являются квазистационарными, поскольку потенциал смещенной решетки с одной из сторон меньше энергии большинства состояний, и энергия состояния имеет комплексное значение с некоторым малым показателем затухания: $E=E_{\text {real }}+i \Gamma$. Наши исследования показали, что пренебрежение затуханием вносит ошибку, заметную до такой степени, что волновые функции соседних состояний перестают быть трансляционно-симметричными. Основная причина этого состоит в том, что при вычислениях помимо „истинной“ ШЛ возникают также паразитные уровни нелокализованных состояний. Показатели затухания, т. е. мнимые части „истинных“ энергий, значительно меньше, чем у паразитных, что и дает возможность их отфильтровать. Таким образом, для точного численного метода необходим способ поиска комплексных корней некоторого нелинейного уравнения. Данное уравнение составляется при помощи метода матриц переноса. Для кусочно-линейного потенциала, которым обладает смещенная сверхрешетка, матрицы переноса должны быть выражены через комплексные функции Эйри, вычисление которых трудоемко. Вместо этого кусочнолинейный потенциал можно приближенно представить в виде кусочно-постоянного (дискретизировать) и использовать матрицы переноса для волновых функций в слое с постоянным потенциалом, выражаемые суммой комплексных экспонент [8]. Такая дискретизация обоснована далее.

Уравнение для поиска энергии $E$ вида $f(E)=0$ выводится из того, что в матрице переноса через всю структуру (она является произведением матриц для отдельных слоев) один из элементов должен быть равен 

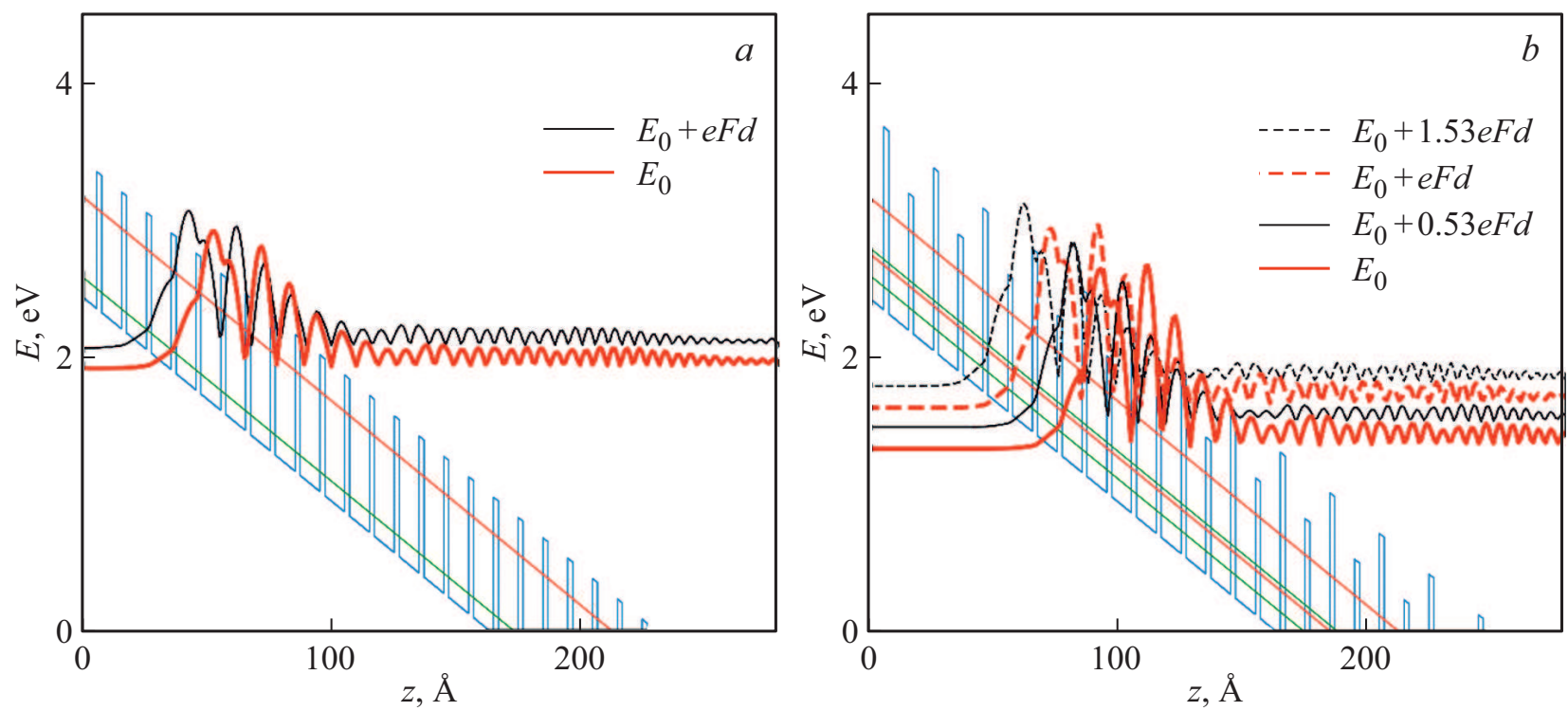

Рис. 1. Трансляционно-симметричные волновые функции в электрически смещенной сверхрешетке. $a-$ для сверхрешетки с немодулированным потенциалом; $b$ - для сверхрешетки с модулированным потенциалом, т. е. со щелью в минизоне. Наклонные линии обозначают границы минизоны (минизон).
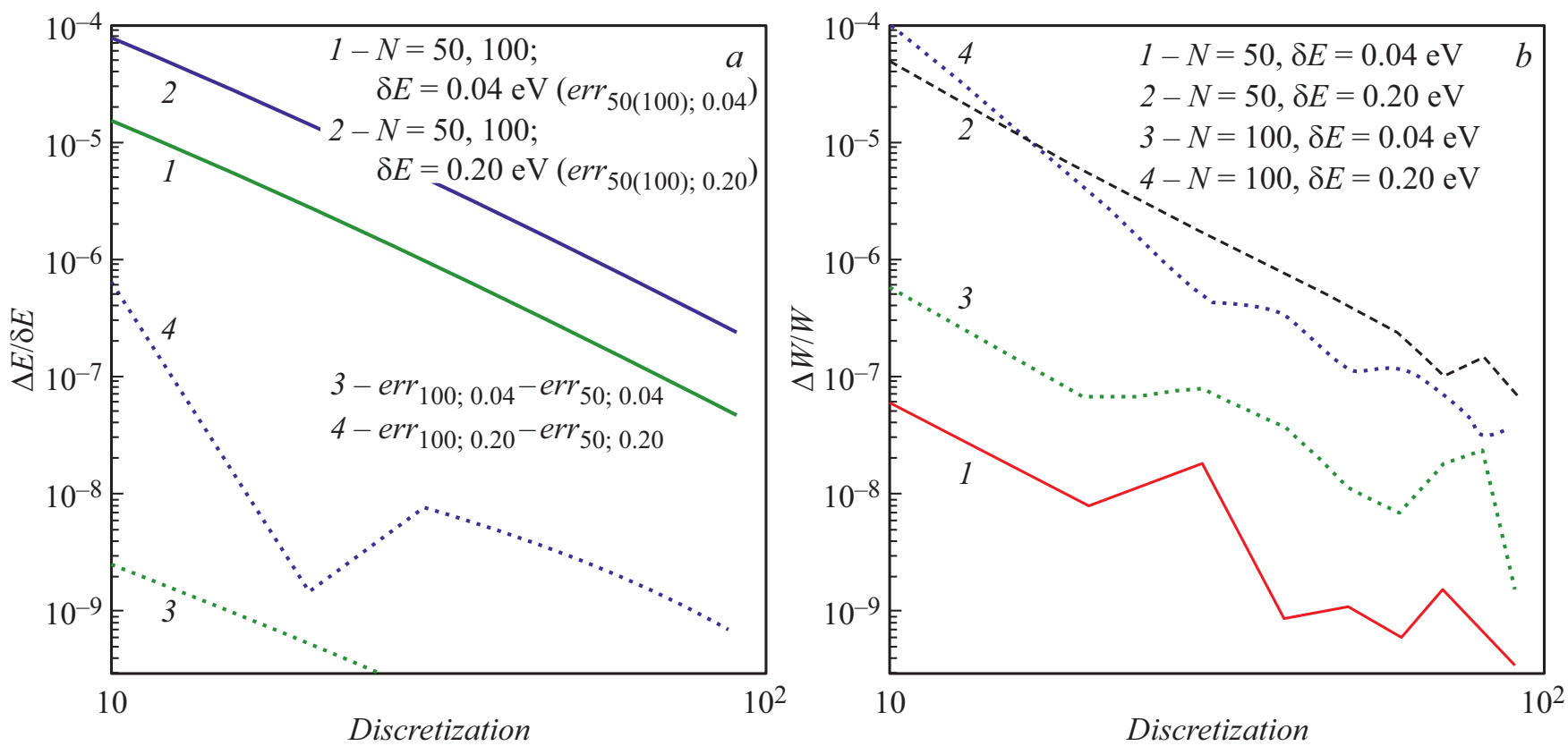

Рис. 2. Влияние количества элементов в представлении линейного участка (барьера или ямы с приложенным смещением) кусочнопостоянной функцией. Зависимость изменения разности последовательных приближений при увеличении дискретизации: $a-$ для величин энергии, отнесенной к расстоянию в ШЛ; $b$ - для величин вероятности излучательного перехода, отнесенной к самой вероятности.

нулю для того, чтобы волновая функция не возрастала экспоненциально при удалении в обе стороны от структуры. Проблема поиска комплексных корней функции $f$ заключается в том, что ее модуль имеет чрезвычайно большую величину - до $10^{100}$ (отметим, что он безразмерен), поэтому методы градиентного спуска неприменимы. Кроме того, нельзя также определить начальные приближения для этих корней и их общее количество. С этими недостатками в общем случае должен справиться метод, основанный на интегрировании по контуру [9]; данный контур может быть прямоугольным на комплексной плоскости со сторонами, параллельными осям. Границы его по вещественной оси выбираются произвольно (исходя из исследуемой 

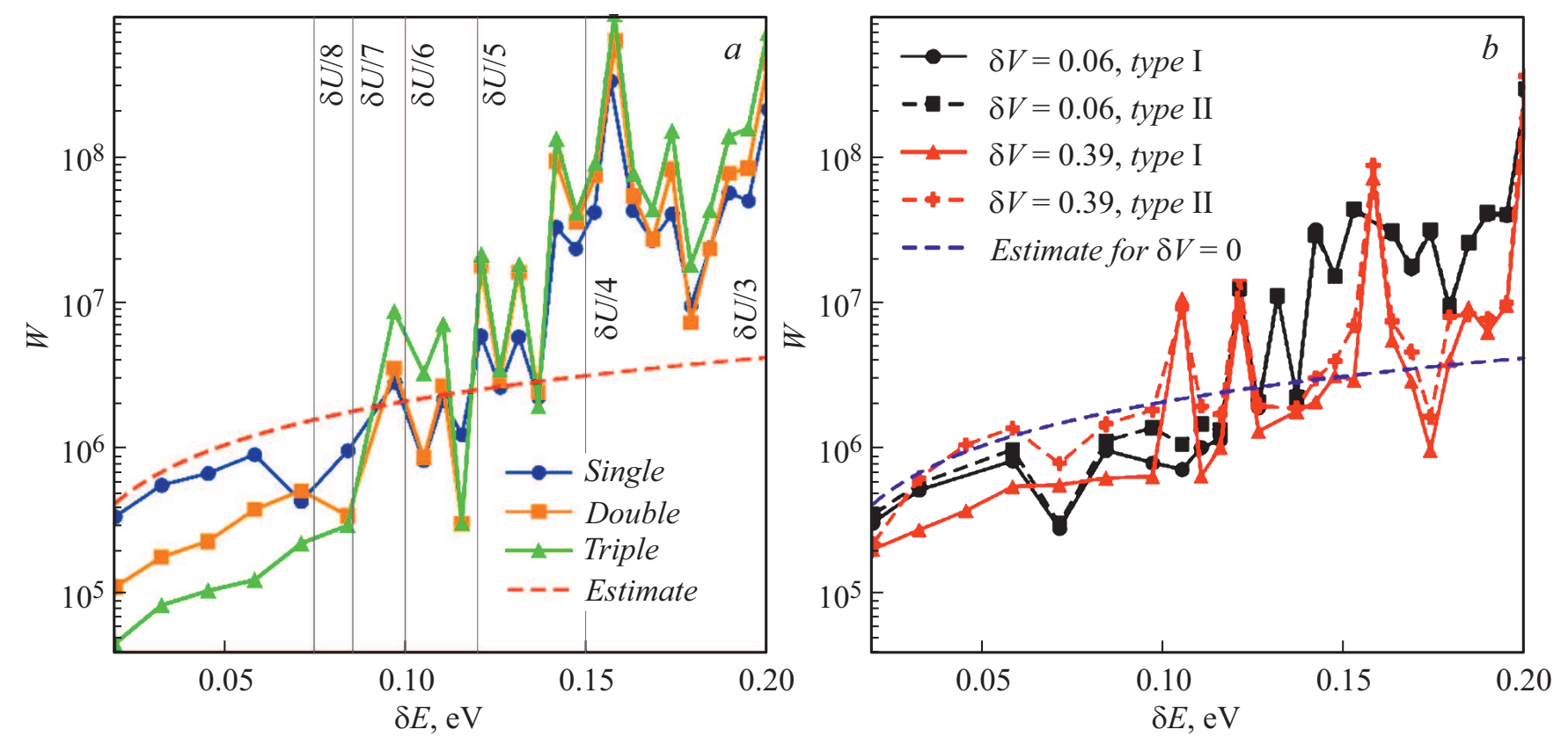

Рис. 3. Зависимость вероятности излучательных переходов от расстояния между соседними уровнями ШЛ, а также оценка вероятности перехода на блоховской частоте (Estimate) согласно (1). $a$ - для сверхрешетки без модуляции потенциала вычислены вероятности переходов на одинарной, двойной и тройной блоховской частоте; $b$ - для сверхрешеток с модуляцией потенциала $\delta V$ вычислены вероятности переходов типа I (частота $\left.\omega_{\mathrm{B}}-\delta \omega\right)$ и типа II (частота $\left.\omega_{\mathrm{B}}+\delta \omega\right)$ при различной величине модуляции, величина $\delta E$ на оси и в оценке рассчитывается так, как будто модуляции нет, для упрощения сравнения с данными, приведенными на части $a$.

области энергий), а границы по мнимой оси - из физических соображений. Однако ошибка, которая накапливается в данном случае в ходе интегрирования из-за амплитуды $|f(E)|$, также не дает возможности им пользоваться.

В ходе исследований оказалось, что аргумент функции $\arg f(E)$ меняется плавно, что позволяет пользоваться для изоляции корней принципом аргумента, известным из теории функции комплексного переменного, который гласит, что для аналитической функции число корней $N$ связано с изменением ее аргумента при обходе по замкнутому контуру выражением $\Delta \arg f=2 \pi N$. Поскольку при численных расчетах аргумент функции находится в области $[-\pi, \pi]$, для нахождения вариации аргумента необходимо найти такие точки контура, где он резко меняет значение с $\pi$ на $-\pi$, и вычесть из их количества число точек, где аргумент меняется в обратную сторону. Поскольку $f(E)$ не имеет полюсов, это число в точности совпадает с количеством корней, а число указанных точек не превышает на практике удвоенного количества корней, так как аргумент меняет ся плавно. Это дает возможность разбивать контур при помощи адаптивного алгоритма, который состоит в следующем.

1. Контур разбивается на равные отрезки, общее количество которых несколько больше числа корней; в точках, разделяющих отрезки, вычисляется $\arg f(E)$.
2. Те отрезки, на которых $\varepsilon<|\Delta \arg f(E)|<2 \pi-\varepsilon$ ( $\varepsilon$ есть некоторое малое число), вновь делятся на равные отрезки, и для точек внутри них данный шаг повторяется.

3. В конце остаются только отрезки, для которых либо $\varepsilon>|\Delta \arg f(E)|$, либо $\Delta \arg f(E)>2 \pi-\varepsilon$ (их число $K)$, либо $\Delta \arg f(E)<-2 \pi+\varepsilon$ (их число $M)$; в результате число корней в контуре $N=K-M$.

4. Если $N>1$ и контур по своим размерам больше заранее заданной погрешности, то он разбивается на четыре новых, для которых шаги 1-3 повторяются.

В результате мы имеем набор контуров, каждый из которых содержит по одному корню и при этом ограничивает сколь угодно малую область. Представленный адаптивный метод эффективен благодаря тому, что в нем значение функции $f$ вычисляется только в тех регионах, где аргумент меняется резко, что позволяет не задавать дискретизацию контура заранее и при этом не опасаться, что какие-то точки скачка аргумента будут потеряны.

Данный метод был использован для вычисления волновых функции локализованных состояний ШЛ в сверхрешетках из 100 периодов (эффективная масса электрона бралась везде $0.5 m_{e}$ ). В одном случае каждый из периодов $d=1 \mathrm{~nm}$ имел яму толщиной $0.8 \mathrm{~nm}$ и барьер толщиной $0.2 \mathrm{~nm}$ и высотой $1 \mathrm{eV}$, что дает минизону $\delta U \approx 0.4 \mathrm{eV}$. В таких сверхрешетках вычислялись вероятности излучательных переходов между соседними уровнями, а также с пропуском одного и двух уровней 
(на частотах соответственно $\omega_{\mathrm{B}}, 2 \omega_{\mathrm{B}}, 3 \omega_{\mathrm{B}}$ ). В другом случае высота каждого второго барьера имела значение $1+\delta V[\mathrm{eV}]$, где $\delta V$ варьировалось от 0 до $0.4 \mathrm{eV}$; это приводит к открытию щели в минизоне, размер которой $\delta u$ доходит до $0.05 \mathrm{eV}$ и растет практически линейно с ростом $\delta V$. Сверхрешетки второго типа использовались, в частности, в работах $[5,6]$ для объяснения ангармонических свойств блоховских осцилляций. В таких сверхрешетках возможны два типа переходов: на частотах $\omega_{\mathrm{B}}-\delta \omega$ и $\omega_{\mathrm{B}}+\delta \omega$, где $\delta \omega$ мало. Мы обозначаем эти переходы соответственно как тип I и тип II. Вероятности излучательных переходов вычислялись по правилу Ферми, причем в простой сверхрешетке для переходов на основной частоте эту вероятность можно оценить [10] (обозначая $\alpha=1 / 137, n-$ показатель преломления) как

$$
W \approx \frac{\alpha}{16 \pi}\left(\frac{n d \delta U}{c \hbar}\right)^{2} \omega_{\mathrm{B}} .
$$

Прикладываемое электрическое поле варьировалось от $2 \cdot 10^{5}$ до $2 \cdot 10^{6} \mathrm{~V} / \mathrm{cm}$, что эквивалентно диапазону $\omega_{\mathrm{B}}$ от 5 до $50 \mathrm{THz}$ или $\delta E$ от 0.02 до $0.2 \mathrm{eV}$.

Результаты вычислений волновых функций приведены на рис. 1. Наблюдаемая штарковская локализация состояний подтверждает корректность метода. Видно, что в сверхрешетке с расщепленной минизоной соседние состояния перестают быть трансляционно-симметричными (это объясняется тем, что настоящий период решетки теперь вдвое больше), однако по форме они по-прежнему близки.

Для выяснения влияния дискретизации кусочнолинейного потенциала вычисления энергий и вероятностей переходов были выполнены для различного числа разбиений одного линейного отрезка на отрезки с постоянной энергией: от 10 до 100 разбиений. Мерой ошибки служило отношение разницы величин при увеличении разбиения к самой величине (этот метод является стандартным в вычислительной физике, так как точное значение величины неизвестно); результаты приведены на рис. 2. Видно, что повышение дискретизации выше 30 не ведет к сколько-нибудь заметному повышению точности. Вычисление вероятности оптических переходов подвержено большей численной ошибке (что видно по хаотическому характеру зависимости на рис. 2, $b$ ), поэтому для основной массы вычислений использовалось значение дискретизации 30.

Наиболее важные с точки зрения физики результаты исследования вероятностей излучательных переходов представлены на рис. 3. Уже для простой сверхрешетки без модуляции потенциала результат отличается от предсказаний и сильно отходит от оценки (1), которая верна лишь для малых смещений (частот переходов). В частности, видно, что при достаточно большом приложенном смещении переходы на кратных частотах могут превышать по вероятности переходы на основной частоте и зависимость вероятностей всех переходов от приложенного поля (или эквивалентно от расстояния между уровнями $\delta E$ ) нелинейна. В ней встречаются как пики, так и провалы. Полной теории, объясняющей такой эффект, нет, однако такое поведение, возможно, качественно объясняется отражением электрона от границ зон Бриллюэна высших порядков, так как пики встречаются вблизи значений $\delta E=p \delta U / q$, где $p$ и $q$ целые, т.е. отношение $\delta E / \delta U-$ рациональное число. Для сверхрешетки с модуляцией потенциала $\delta V$ нелинейность сохраняется, но появляются и новые эффекты. В то время как при малой модуляции переходы типов I и II по вероятности слабо отличаются друг от друга и от переходов в решетке с $\delta V=0$ (что физически корректно), при увеличении $\delta V$ тип II доминирует над типом I. Кроме того, некоторые пики зависимости $W(\delta E)$ инвертируются.

Таким образом, в работе представлен быстрый и надежный метод моделирования волновых функций носителей в электрически смещенных одномерных периодических структурах, с его помощью исследованы ангармонические блоховские осцилляции и продемонстрирована их нелинейная природа при величине расстояния между уровнями в штарковской лестнице, приближающейся к ширине минизоны сверхрешетки.

\section{Финансирование работы}

Работа выполнена в рамках государственного задания по теме 0791-2020-0002 FSRM-2020-0002 „Создание и исследование новых типов светоизлучающих приборов и структур на основе одномерных и трехмерных микрорезонаторов с квантово-размерной активной областью“ и поддержана грантом Российского фонда фундаментальных исследований № 18-32-00801.

\section{Конфликт интересов}

Авторы заявляют, что у них нет конфликта интересов.

\section{Список литературы}

[1] C. Zener, Proc. Roy. Soc. Lond. A, 145 (855), 523 (1934).

[2] G.H. Wannier, Phys. Rev., 117 (2), 432 (1960). https://dx.doi.org/10.1103/physrev.117.432

[3] L. Esaki, R.Tsu, IBM J. Res. Dev., 14 (1), 61 (1970). https://dx.doi.org/10.1147/rd.141.0061

[4] J. Stockhofe, P. Schmelcher, Phys. Rev. A, 91 (2), 023606 (2015). https://dx.doi.org/10.1103/PhysRevA.91.023606

[5] V.I. Sankin, A.V. Andrianov, A.O. Zakharin, A.G. Petrov, Appl. Phys. Lett., 100 (11), 111109 (2012). https://dx.doi.org/10.1063/1.3693418

[6] K.A. Ivanov, A.G. Petrov, M.A. Kaliteevskii, A.J. Gallant, Письма в ЖЭТФ, 102 (12), 911 (2015). https://dx.doi.org/10.1134/s0021364015240054

[7] D. Tamascelli, S. Olivares, S. Rossotti, R. Osellame, M.G.A. Paris, Sci. Rep., 6 (1), 26054 (2016). https://dx.doi.org/10.1038/srep26054 
[8] Л.Е. Воробьёв, Е.Л. Ивченко, Д.А. Фирсов, В.А. Шалыгин, Оптические свойства наноструктур (Наука, СПб., 2001).

[9] P. Kravanja, M. Van Barel, Computing the zeros of analytic functions (Springer, 2007). https://dx.doi.org/10.1007/bfb0103927

[10] A.M. Bouchard, M. Luban, Phys. Rev. B, 52 (7), 5105 (1995). https://dx.doi.org/10.1103/PhysRevB.52.5105 